\title{
Wenn man nicht mal mehr lachen kann ...
}

\section{Samia Hurst}

Prof. Dr. med., Institut Ethique, Histoire, Humanités (iEH2), Faculté de Médecine, Genf

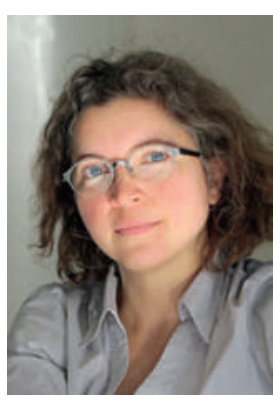

1 Watson K. Gallows humor in medicine. Hastings Cent Rep. 2011;41(5):37-45. Pub Med PMID: 21980898.

2 Parsons GN, Kinsman SB, Bosk CL, Sankar P, Ubel PA. Between two worlds: medical student perceptions of humor and slang in the hospital setting. J Gen Intern Med.

2001;16(8):544-9. PubMed PMID: 11556931. Pubmed Central PMCID: 1495252.

3 Anonymous. Our Family Secrets. Ann Intern Med. 2015;163(4):321.

4 Laine C, Taichman DB, LaCombe MA. On Being a Doctor: Shining a Light on the Dark Side. Ann Intern Med. 2015;163(4):320. PubMed PMID: 26280419. Wear D, Aultman JM, Varley JD, Zarconi J. Making fun of patients: medical students' perceptions and use of derogatory and cynical humor in clinical settings. Academic medicine: journal of the Association of American Medical Colleges. 2006;81(5):454-62. PubMed PMID: 16639201.

samia.hurst[at]saez.ch
Diese Anekdote beruht auf einem tatsächlichen Ereignis. Wir befinden uns in einer Notfallabteilung in den USA. Ein paar hungrige Assistenzärzte warteten auf die gerade bestellte Pizza, als die Ankunft eines Patienten mit Schussverletzung angekündigt wird. Sie tun alles, um das Leben des schwerverletzten Opfers zu retten - vergeblich. Es war der Pizzabote, der vor dem Spital angeschossen wurde. Nachdem wieder Ruhe eingekehrt ist, finden die Ärzte die Pizza auf der Türschwelle, immer noch in appetitlichem Zustand. Als sie die Schachtel öffnen, fragt einer: «Was meint ihr, wie viel Trinkgeld sollten wir ihm geben?» Alle lachen und essen die Pizza [1]. Medizinischer Humor gibt es in den verschiedensten Formen und kann für Aussenstehende, die ihm das erste Mal begegnen, schockierend sein. Generationen von Praktikanten haben diese Diskrepanz erlebt [2]. Wir schulden unseren Patienten hohen Respekt und absolute Höflichkeit. Was also sollen wir von den manchmal sehr groben Witzen halten, die unter uns ausgetauscht werden? Die naheliegendste Antwort: Dieser Humor ist ein wichtiges Ventil, eine Art, die Last des menschlichen Leidens, das uns anvertraut wird, erträglicher zu machen. Wer am lautesten lacht, ist oft auch derjenige, der durch das Schicksal seiner Patienten am meisten berührt ist.

So notwendig und legitim er ist, so hat der medizinische Humor doch auch eine dunklere Seite. Dieses Mal befinden wir uns in einem Gebärsaal, wiederum in den USA, wo der Geburtshelfer gerade dank einer Massage der Gebärmutter unter Narkose das Leben einer Frau spanischer Herkunft retten konnte, die nach der Geburt zu verbluten drohte. Erleichtert und glücklich, die eine Hand noch in der Vagina, hob er die andere Hand, begann zu tanzen und sang dazu «La Cucaracha, la cucaracha ...». Hier stoppte ihn der Anästhesist sofort. Diese Geschichte wurde kürzlich anonym publiziert [3], einige Jahre später, mit geradezu greifbarer Scham. Die Redaktoren der Zeitschrift hatten lange darüber verhandelt, ob die Geschichte veröffentlicht werden sollte. Sie erläutern dies im Editorial und es scheint, als ob sie sich ebenfalls dafür schämten [4]. Eine Grenze war überschritten worden.

Diese Grenzen existieren also. Wo aber liegen sie, und wie können wir sie einhalten, ohne gleich in eine Art
Moralismus zu verfallen, der jeglichen Humor verbietet? Die Juristin Katie Watson hat eine Feinanalyse zur Kategorisierung des Humors nach seinen Funktionen erstellt [1]. Zwischen den Zeilen unserer Spässe und Witze verstecken sich alle Arten von Botschaften, und sie sind es, die den Unterschied ausmachen. Wir scherzen, um einem Kollegen eine Wahrheit schneller sagen zu können. Weil es wirkungsvoll ist, verwenden wir dies oft als rhetorisches Mittel, um eine Kritik abzutun. Diese Art Humor ist schon etwas zweifelhafter. Wir benutzen oft Humor, um über etwas zu lachen, das eine Macht über uns ausübt, um so eine Situation herzustellen, in der ein sonst unerträgliches Ungleichgewicht ausgehalten werden kann. Die Ziele des schwarzen Humors sind hier die Krankheit, die Not oder, wie in der Geschichte mit dem Pizzaboten, sogar der Tod. Dieser Humor ist notwendig, lebenswichtig. Er erlaubt uns zu überleben, zu lächeln

Wer am lautesten lacht, ist oft auch derjenige, der durch das Schicksal seiner Patienten am meisten berührt ist.

und (manchmal direkt danach) den nächsten Patienten zu behandeln. Für diese Art des Humors brauchen wir uns nicht zu schämen. Wir benutzen Humor, um uns mit unseren Mitmenschen solidarisch zu zeigen, um unseren Schmerz zu teilen.

Wir können Humor aber auch benutzen, um uns abzugrenzen. Um zu betonen, an welchem Punkt wir uns von den Patienten unterscheiden, um sie als Verursacher der Schmerzen zu sehen, die sie uns bereiten, wenn wir ihnen nicht besser helfen können [5]. Diese Art Humor trifft diejenigen, die schwächer sind als wir, und ja, dies kann Probleme verursachen.

Katie Watson bietet hier ein Hilfsmittel an: Wer ist das Ziel des Scherzes? Könnte das Scherzen die Qualität unserer Behandlung einschränken? Könnte es zu schnell eine Erleichterung bringen und uns dabei die Motivation nehmen, eine inakzeptable Situation zu verändern? Wer hört zu und könnte unter dem Scherz leiden? Ein nützliches Hilfsmittel, das hilft zu verstehen, wo die Grenzen sind, ohne uns das Scherzen und letztlich das Lachen zu verbieten. 